\title{
Erratum to: Parameters related to a positive test result for FDG PET(/CT) for large vessel vasculitis: a multicenter retrospective study
}

\author{
G. A. Hooisma $\cdot$ H. Balink $・$ P. M. Houtman • \\ R. H. J. A. Slart • K. D. F. Lensen
}

Published online: 24 March 2012

(C) Clinical Rheumatology 2012

\section{Erratum to: Clin Rheumatol \\ DOI 10.1007/s10067-012-1945-0}

In the version of the article initially published, the figures do not correspond to its captions. Here are the figures with correct captions.

The online version of the original article can be found at http://dx.doi. org/10.1007/s10067-012-1945-0.

G. A. Hooisma $\cdot$ H. Balink $(\bowtie)$

Department of Nuclear Medicine, Medical Center Leeuwarden,

Leeuwarden, The Netherlands

e-mail: hans.balink@znb.nl

K. D. F. Lensen

Department of Internal Medicine, Institute for Cardiovascular

Research, VU University Medical Center,

Amsterdam, The Netherlands

\section{P. M. Houtman}

Department of Rheumatology, Medical Center Leeuwarden,

Leeuwarden, The Netherlands

\section{R. H. J. A. Slart}

Department of Nuclear Medicine,

University Medical Center Groningen,

Groningen, The Netherlands 
Fig. 1 Left image is a

maximum intensity projection

(MIP) of the PET investigation, showing pathological uptake in the carotids and bilateral subclavia. SUV max 2.4 Discrete increased uptake in thoracic aorta. The patchy uptake, with low intensity, in the abdominal aorta is mainly due to atherosclerosis seen on the coronal CT slice. Right image is a coronal CT slice of the abdominal aorta

Fig. 2 Left image is a maximum intensity projection of the PET investigation, showing pathological activity in aortic arch and abdominal aorta until the bifurcation, and in both subclavian arteries and art. brachialis on both sides. SUV max 4.3. Right image is a transverse CT slice showing wall thickening of the aorta at the level of the crus of the diaphragm
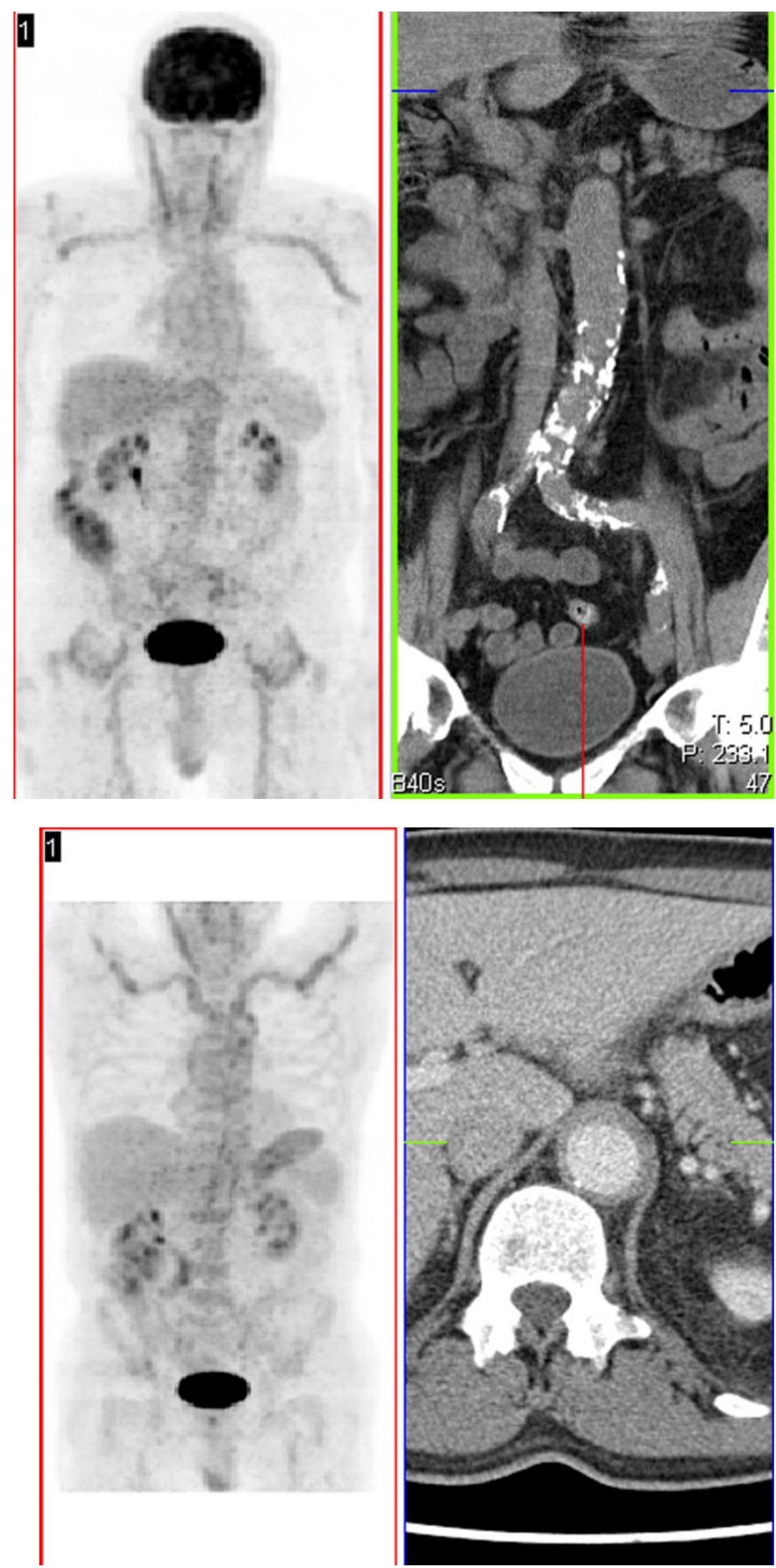
Fig. 3 Left image is a maximum intensity projection of the PET investigation; pathological activity in aortic arch and abdominal aorta until the bifurcation, and in the subclavian, brachial, and carotic arteries. SUV max 5.4. Right image is a transverse CT slice; no wall thickening in thoracic and abdominal aorta
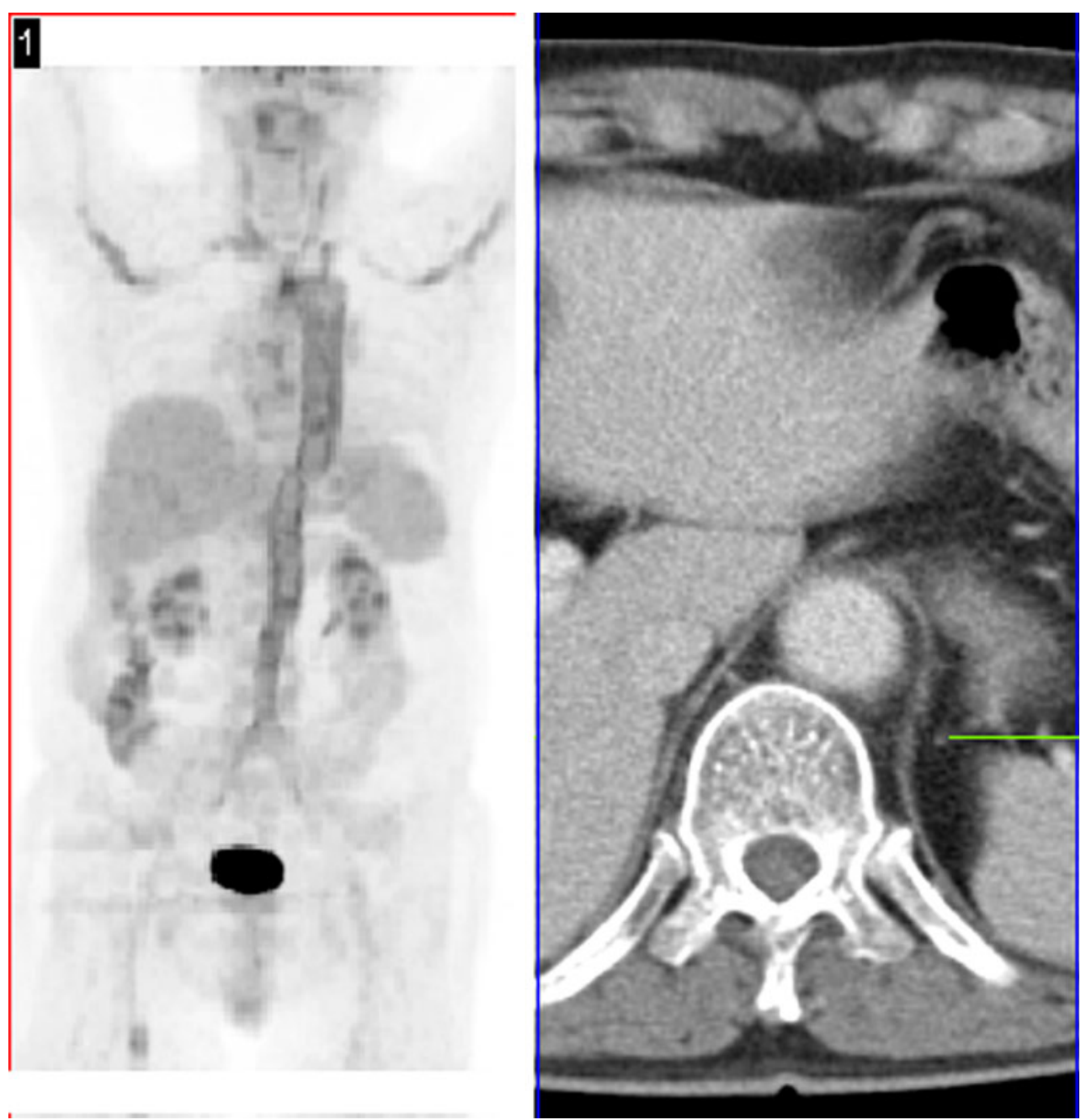